\title{
LA PRODUCCIÓN DE CONOCIMIENTO: ALGO SABIDO Y NO CORREGIDO
}

\section{Blanca Pascual Ortigosa}

Investigadora independiente

\section{Resumen}

El objeto del presente artículo es desarrollar una aproximación a los retos contemporáneos a los que debemos enfrentarnos desde el punto de vista de la articulación historiográfica.

Para esta investigación, resulta inevitable como proceso metodológico, retrotraernos al análisis de la producción de conocimiento. Partiremos del exámen del propio término "Arte Feminista" y continuaremos revisando, para estudiar la vigencia de éstos, los mecanismos, cánones y sistemas de legitimidad que erigen dicha producción. Procesos, que como el propio título indica, han sido investigados por numerosas críticas y teóricas de Arte Feminista, desde la década de los 70 hasta la actualidad, pero que no encontramos superados y/o corregidos por propuestas efectivamente renovadoras dentro de los espacios hegemónicamente legitimados. En este escenario, otros debates residuo de esta brecha entre la vida y la norma, adquieren presencia en estos días bajo diferentes signos. Como otro más de los desafíos que este artículo despliega, los propias herramientas, estrategias y metodologías, deberán ser examinadas desde una perspectiva feminista.

\section{Palabras clave: PRODUCCIÓN DE CONOCIMIENTO; CANON; LEGITIMI- DAD; DESAFÍOS}

\section{THE PRODUCTION OF KNOWLEDGE: SOMETHING KNOWN BUT NOT CORRECTED}

\begin{abstract}
:
The purpose of the present article is to develop an approximation to the contemporary challenges we must face from the historiographical articulation point of view.

For this investigation, it is unavoidable to go back to the analysis of the production of knowledge, as a methodological process. We will start studying the term "Feminist Art" and we will continue reviewing the mechanisms, canons and legitimacy systems that construct the production of knowledge, in order to analyze the validity of these. Processes that, as the title indicates, have been investigated by numerous critics and theorists of the Feminist Art, from the 70's decade until nowadays, but that we do not yet find left behind or corrected by effective innovative proposals within the legitimized hegemonic spaces. In this scenario, other remnant debates of this gap between life and rulegain presencetoday under different signs. Like yet another of the challenges that this article unfolds, strategies and methodologies should be examined from a feminist perspective.
\end{abstract}

Keywords: PRODUCTION OF KNOWLEDGE; CANONS; LEGITIMACY; CHALLENGES

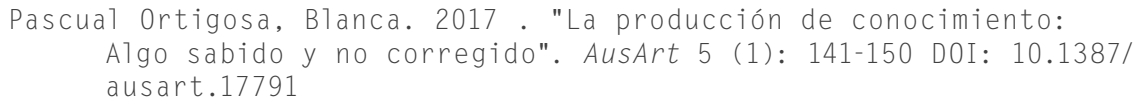

\section{AUSART}




\section{A VUELTAS CON LA CONSTRUCCIÓN DE LA HISTORIA}

El campo de estudio que en la que esta investigación se sitúa tiene que ver con la propia construcción -y sus límites- del marco de análisis que despliega este número AUSART. Aunque las premisas de partida son sabidas, merece la pena revisitarlas y atender así a la importancia de los procedimientos con el objeto de acercarnos a las experiencias actuales.

Analizar la problemática, y por qué no decir, la injusticia, que platea el término "Arte Feminista" será una buena estrategia para ubicarnos. Esta "categoría" ha sido el movimiento más extenso y con un mayor volumen de experiencias artísticas desde la II Guerra Mundial, englobando un amplísimo rango de prácticas, medios o discursos, muchas de ellas entroncándose con lo que conocemos como prácticas postmodernas y disruptivas.

Mientras otros movimientos han sido conceptualizados por conexiones o aproximaciones en tendencias conceptuales, estilísticas, materiales o geografías, este movimiento ha sido definido por su afinidad -por ser una respuesta desde las prácticas artísticas- a un movimiento social y político, con independencia de sus ramas, disyuntivas, perspectivas o extensión en el tiempo. Parece, por tanto, insólito pensar bajo una misma categoría-paraguas tal inestabilidad formal, tan amplio registro de discursos artísticos -a veces en tensas oposiciones, o incluso contradicciones-, medios o geolocalizaciones.

De manera paralela, Griselda Pollock nos alerta de la sensibilidad de los procesos de construcción en términos de feminismos, pues éstos permanecen en continua transformación y revisión. "Todavía nos estamos convirtiendo en feministas. (El feminismo) No es sabido y todavía no es acabado, el feminismo es "virtual", una promesa"(Pollock 2008, 59).

Por lo tanto, y dado que conformar una categoría implicaría acotar temporalmente, definir maestras, movimientos, discursos, estilos, etc., deberíamos poner sobre la mesa la cuestión de cómo crear - y si estaríamos interesadasunos patrones, o al menos las ideas-fuerza, de un cuerpo blando, permeable, no jerárquico y en continuo movimiento.

La estrategia de Lourdes Méndez propone pensar la categoría de "Arte feminista" como un "modelo reducido", haciendo así mismo referencia a la con- 
ceptualización de Lévi-Strauss, que nos permitiría observar conexiones entre obras y paralelismos entre culturas y así establecer un marco de estudio, que de otro modo quizás hubiese pasado desapercibido. Es decir, lo que nos plantea la autora es que estaríamos constituyendo una "etiqueta ficticia" pero que nos permite contextualizar y elaborar una historia compleja.

Acordando esta primera racionalización de la terminología- por otro lado, nada nueva, como ya anunciamos en el título-, y para apuntar a cómo se sitúa este movimiento en las reivindicaciones, convendría atender, y desmontar, dicho sea de paso, la popular creencia -en la que se instalan incluso hoy en día muchas propuestas museísticas- de que nos interesa rescatar artistas por el hecho de ser mujeres invisibilidades por la historia del arte.

Este desvío, además ser complejo por la pluralidad del sujeto que nombra de manera unificada, resulta de especial peligrosidad por las cuestiones que evita en su atajo. Sin embargo, si lo que nos interesasefuese revisar trayectorias porque con sus prácticas han sido precursoras de paradigmas artísticos y han incidido y transformado radicalmente la historia del arte, y sin estos eslabones creadores de vanguardia, la historia hubiese sido otra, esto significaría situar el campo de estudio en los mecanismos que han articulado, sostenido y legitimado este soterramiento.

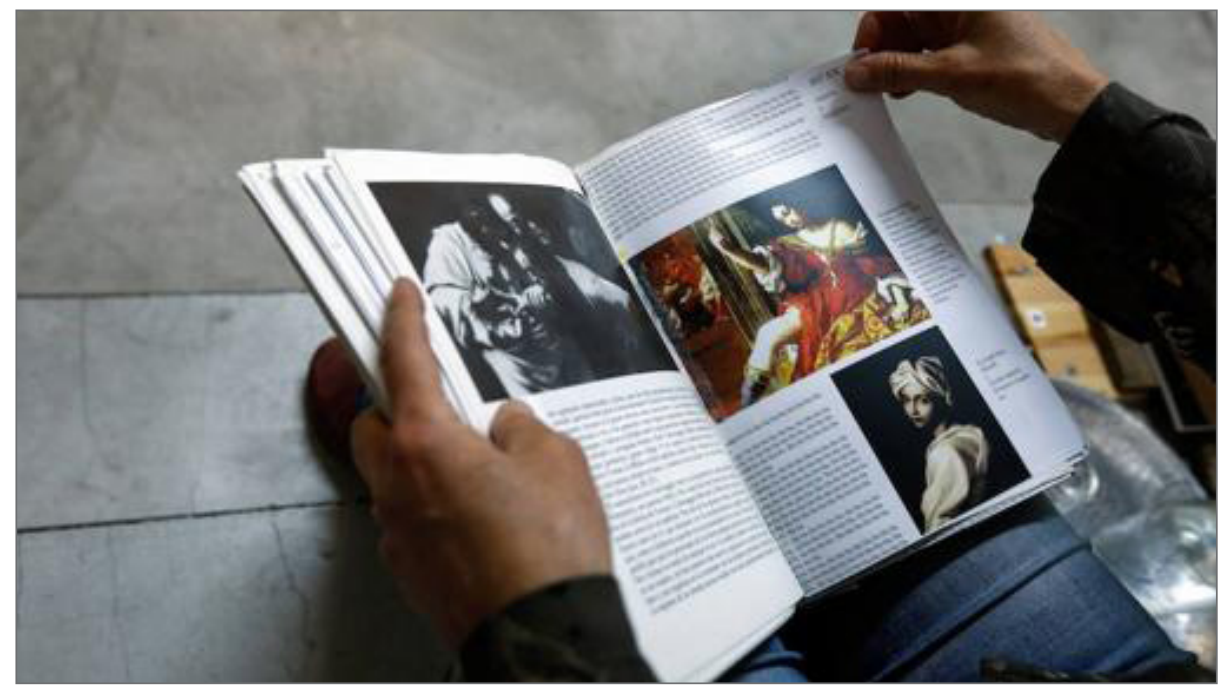

"Queridas Viejas". María Gimeno (2017) Performance en la que intenta corregir la ausencia de mujeres en los manuales de historia. 
Artistas que han habilitado caminos, han revolucionado la idea de arte, y han cuestionado no solo los cánones heteropatriarcales, sino los propios procesos artísticos, no aparecen citadas en la narración de la Historia del Arte. Dado este estado de la cuestión, las investigaciones se dirigen a revisar las estructuras que sustentan la construcción historiográfica.

$Y$ de nuevo, este ejercicio no responde un asunto de reclamo justiciero. Si deseamos revisar estos pilares se debe a que, parafraseando a Pollock, la relación que creamos con el pasado determina cómo habitamos el presente $\mathrm{y}$, sobre todo, cómo los imaginarios colectivos construyen el futuro. Activar genealogías de revolucionarias precursoras, supone activar un futuro común.

El control de la cultura ha sido fundamental en todas las épocas y civilizaciones por su relación con la capacidad de ejercer poder. Por ello T. J. Clark apunta "la cultura no es sino un campo de batalla de representaciones, dado que únicamente conocemos el mundo a través de las representaciones que de él se nos presentan"(Pollock 2007, 23). Por tanto, el control de las representaciones significa el control de las narraciones historiográficas y de las identidades colectivas.

Estaremos situando la mirada si a ello añadimos la afirmación de Laura Cottingham "el sexismo ha deformado todos los aspectos del arte: desde la teoría sobre la historia del arte hasta la economía del mercado del arte"(Phelan 2001, 19). Pollock completa la referencia crítica señalando que:

"La historia del arte ha sido configurada y sostenida por ideologías patriarcales: grandes obras de arte producidas por los grandes hombres de cada nación, que reflejan e instruyen a cada nación y a cada cultura sobre cuáles son sus ideas más brillantes (incluyendo jerarquizaciones implícitas: periodo, estilo, nación, maestro, técnica, etc.). Es decir, la historia del arte es espejo de las estructuras sociales."

(Pollock 2007, 24)

La metodología conduce a continuación a mirar a los lados de la Institución-Arte, a sus gentes legitimadores. A uno encontraríamos la Academia y al otro el Mercado del arte. Conviene recordar entonces sus cánones y procedimientos, su maquinaria. 
La Academia se vertebra en base a un conjunto de archivos desde los que se articula un corpus de conocimientos. Esta selección de archivos y discursos no es neutral, sino está sustentada en cánones que responden a relaciones de poder y jerarquías, y que constituirán el valor social, estético y artístico. Estos archivos, residuos culturales de la discriminación social, se refuerzan, reproducen y legitiman así mismos por las inercias académicas. En este sentido, Michael Foucault sentencia que el poder del archivo reside en que se convierte en el garante de lo que puede ser dicho.

Pollock $(2008,44)$ conceptualizará, así mismo, la idea de "patriarchivo", que "no solo se institucionaliza como disciplina académica, curatorial y crítica, si no que funciona (además de para respaldar la autoridad) como base imaginaria y soporte mítico." Dispositivo que, como anteriormente aludíamos, pone en peligro además de nuestra construcción de presente, nuestra construcción de futuro.

Teresa de Lauretis amplía el marco identificando la historia del arte en sí misma como una "tecnología de género", pues produce efectos en los cuerpos, en los comportamientos y en las relaciones sociales, interfiriendo, por tanto, en la construcción de la identidad de la persona.

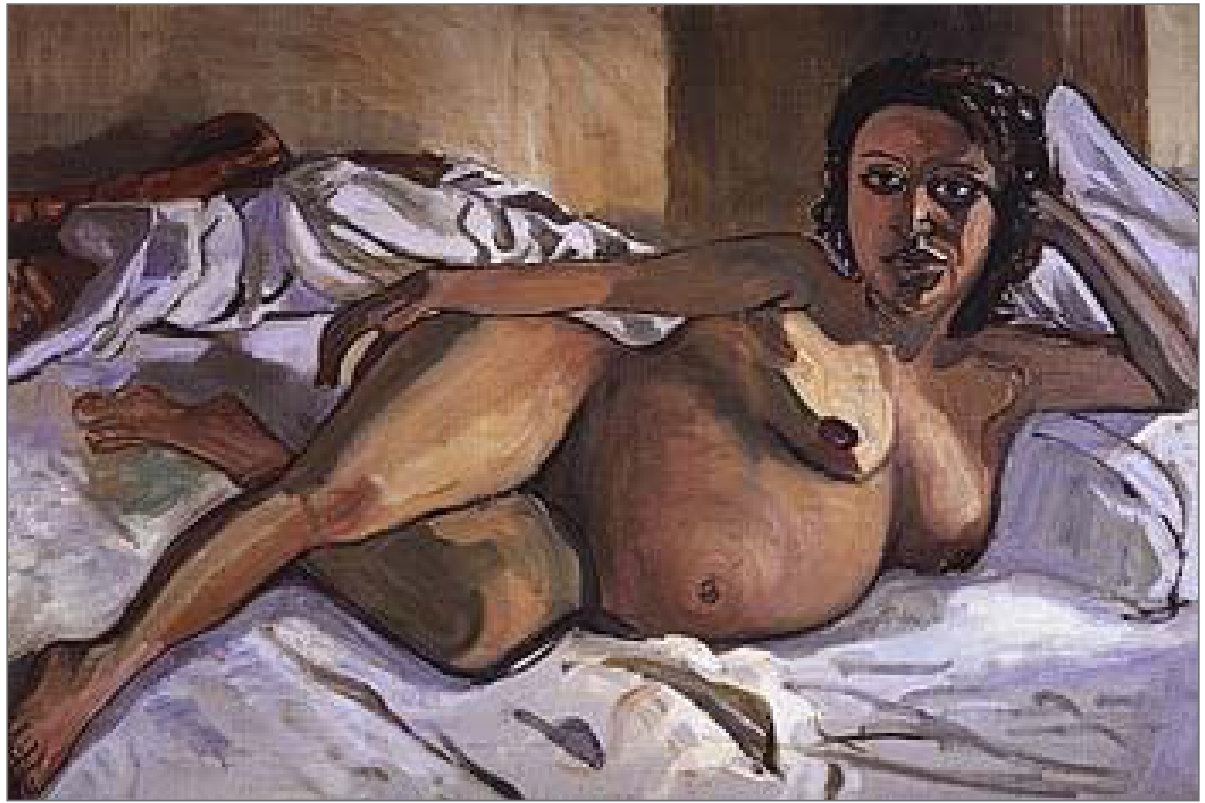

"Pregnant María”. Alice Neel. (1962)

Desafía la expectativa del espectador ante el desnudo. 
Otra de las formas que auspician las estructuras sexistas es el Mercado del Arte. Esta Institución - incluyendo todas sus fórmulas y circuitos de arte- no solo se ha mostrado discriminativa en las direcciones de los flujos de capital, sino que, como consecuencia de ello, ha influido tanto en contenido como en estrategias estéticas, perpetuando la producción de narratividades orientadas a la mirada y gusto de los grandes coleccionistas, tradicionalmente varones, blancos y de clase alta.

Si veíamos que la academia se vigorizaba y legitimaba a sí misma en sus referentes y límites mediante generaciones de investigadores, docentes y estudiantes, reproduciendo y perpetuando estos conocimientos sexistas, lo neurálgico sigue siendo que se configura a sí misma como una vía de difusión extraordinaria exenta de ser cuestionada por agentes ajenos a ella, pues éstos no son reconocidos como legítimos.

Las lógicas de exclusión de las leyes de mercado -fundamentadas en las mismas estructuras- son creadas y legitimadas no por entes no corporales o abstractos y por tanto difíciles de señalar, sino por responsables de espacios museísticos, curadurías y otros agentes de la mediación contemporánea, de los que habría que observar, como otro de los actuales retos, no solamente los procesos de exhibición, sino la compra de obra de artistas, exigiendo, al menos a los espacios de participación pública, la corrección en sus colecciones de la tendencia del mercado privado.

"En España la presencia de mujeres en las colecciones de los museos y centros de arte no llega al 30\%. El Centro Galego de Arte Contemporáneo (CGAC) tiene el mayor porcentaje: un 29 \%. El Museo de Arte Contemporáneo de Castilla y León (Musac) presenta un 23,9\%. En la colección de TEA Tenerife Espacio de las Artes el porcentaje de mujeres artistas asciende a un 21,40\%. En el Guggenheim de Bilbao las autoras representadas en su colección rondan el 17,8\%. La colección permanente del Centro Andaluz de Arte Contemporáneo (CAAC) de Sevilla presenta un 13,9\% de autoras. EI IVAM un 13,8\%, el Museu d'Art Contemporani de Barcelona MACBA el 9,5\% y la colección del Museo Centro de Arte Reina Sofía, según un informe de 2011 de MAV, un 4\% de obras de mujeres y un $6 \%$ de artistas españolas."

(Constenla, 2013) 


\section{FUTUROS NO CORREGIDOS ( $Y$ SUS METODOLOGÍAS)}

Dirigiendo nuestra atención hacia cómo aproximarnos a los retos contemporáneos desde lo ya sabido y no corregido, la idea continúa sin ser la producción de una nueva recopilación irreverente de archivos, sino que el trabajo tendrá como objetivo-entre otros- el dinamitar los cánones falocéntricos que funcionan como lógicas de producción de conocimiento y que impiden que la historia cambie.

En su lugar, el desafío se vincula a un estudio articulado desde una perspectiva multicéntrica (en lo que las luchas de los feminismos negros han tenido tanto que ver), plural, plástica y en continua revisión, que pasa inherentemente por la observación de las posibilidades de construcción del sujeto mujer y las identidades trans o cyborg, reconociendo, y posibilitando, la complejidad de su continua transformación.

Dado que, como expone el artículo de Linda Nochlin, de 1971, "Why have there been no great female artists?" no habrá un reconocimiento de estas creadoras en la historia del arte hasta que no haya condiciones de igualdad social, política, económica en las estructuras políticas y sociales, y como reto parece a tan largo plazo que se nos escapa de las manos, la cuestión sería cómo seguir enunciando, reconociendo y enfrentando los desafíos contemporáneos que habitan nuestro presente en la producción de conocimiento.

Uno de estos reclamos, al igual que los feminismos los extienden en otras luchas, sin duda es el estar y formar parte de los procesos decisorios -no solamente consultivos- y legitimadores. Es decir, se excluye de esta participación decisoria al cuerpo en discusión; en este caso artistas y precursoras. Este mecanismo se conforma como un legado arrastrado de los procedimientos coloniales. Ya que un proceso y una metodología feminista debe situarse políticamente, reconocer luchas yubicarlas herramientas que emplea, tal y como nos indica la artista y activista Daniela Ortiz, es de vital importancia plantearnos si es lícito - o al menos éticamente legítimo- que los países occidentales se apropien de estas herramientas y saberes de los sujetos coloniales y racializados, aun discriminados por los feminismos blancos, pues esto implicaría señalar a la población euroblanca como sujetos faltos de poder. Por ello, resulta imprescindible, cuando hablamos de feminismos, sus metodologías y estrategias, atender estas herramientas de una manera contextualizada. 

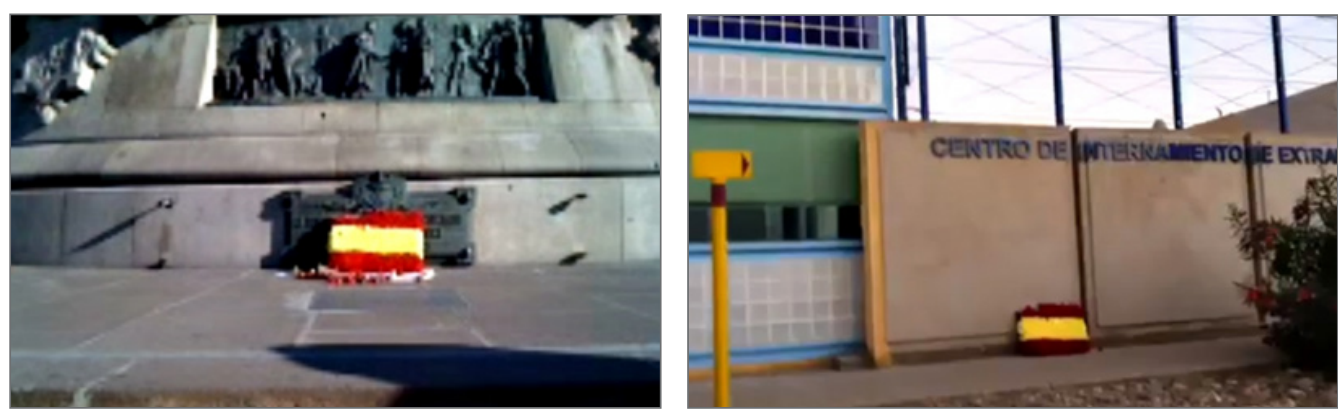

"Ofrenda". Daniela Ortiz. (2012).

El 12 de octubre, retira un arreglo floral del monumento de Colón y lo deposita en el Centro de Detención de Inmigrantes de Barcelona.

De la misma manera, y en un proceso paralelo, la teoría crítica contemporánea deberíareflexionara cerca de la validez de aplicar dispositivos analíticos provenientes del centro anglosajón para el estudio de obras y artistas no europeas o norteamericanas, al menos hasta mediados de la década de los 90, donde podríamos situar esa frontera en la que la globalización de la información hace proliferar los puntos de accesos a internet y por tanto la circulación de la información.

Al hilo de la actual exposición-en el momento en el que se escribe este artículo- en el Museo de Arte Contemporáneo de Vigo, MARCO, "Alén dos xéneros" Prácticas artísticas feministas en Galicia" (del 27 de enero al 11 de junio de 2017), las discusividades y metodologías feministas deberían interrogarse en rigor, entre otras, sobrecuáles son los espacios de investigación que suscita la participación de hombres en exposiciones de arte feminista. Pollock plantea que la obra de una artista no se puede "desfeminizar", por lo que, si suscribiésemos esta premisa, la posibilidad de poder acceder a "lo otro", feminizar, y desprendernos de ello a antojo, se constituiría como privilegio. Aprovechando las posibilidades que esta cuestión nos ofrece, se nos abre un rico campo de estudio para continuar ensayando,para experiencias venideras, estrategias feministas que tengan en cuenta los sistemas de privilegio y su contextualización histórica, y las potencialidadesde posiciones e identidades no binarias.

Entroncando por tanto con nuestras posibilidades y limitaciones como sujetos políticos, convendría no perder de vista la cuestión que ponemos a trabajar continuamente en esta investigación: la cuestión de la legitimidad. Si el sujeto mujer no es agente válido para la producción de conocimiento dados los 
mecanismos excluyentes previamente estudiados, se despliegan preguntas para repensar -de nuevo- estos pilares sobre los que consolidan estos procesos legitimadores, que pasan por plantear cómo alterar esta ecuación desde una lógica no articulada en base al esquema centro-periferia, sino multicéntrica, o si existen dispositivos para pensar, y aplicar, una legitimidad de doble direccionalidad.

Asistimos en el estado español, de una década a esta parte, a prácticas que pretenden estudiar, y habitar, procesos democratizadores y del procomún en la prácticas culturales y producción de saberes, incorporando desarrollos sostenibles, innovaciones situadas y fuentes de conocimiento diverso (obsérvense ejemplos de iniciativas como los Laboratorios Colaborativos, Escuelita CA2M, Zemos, o Colaborabora, entre muchos otros). Previamente habíamos podido observar los intentos por democratizar (siempre manteniendo patrones discriminantes en función de raza, género o clase, resultado de las coetáneas estructuras socializantes) el acceso a la educación. Pero no hemos podido observar aún la incorporación de dispositivos democráticos y transversales -que incorporen la propia metodología del corpus de estudio, en este caso la metodología feminista- en la producción de conocimiento.

La esterilización de artistas revolucionarias en la historia de arte nos ha dejado muchos traumas y procesos no resueltos y/o no conectados, que impregnan la actual cultura. Resquicios de dislocación entre cultura, historia y arte los hayamos desplegados y/o superpuestos en la cotidianeidad de nuestros días; como confusas propuestas expositivas que, si ya resulta complejo homogeneizar bajo el término feminista, se articulan bajouna únicacondición asignada, reincidiendo en asimilarnos como colectivo;o tensas relaciones entre activismos y producción artística, llegando incluso a rechazar cualquier vinculación con el movimiento político aun propuestasque se nutren de investigaciones y estudios feministas.

Los desafíos en la actualidad siguen pasando, además de por auto-autorizarnos una posición legítima que active y respalde dispositivos críticos, por un análisis desde la perspectiva feminista de las herramientas que deseamos emplear para deconstruir los actuales cánones y procesos. Ello con el objeto de generarun conocimiento que tenga en cuenta los diferentes centros, plural y sin exclusiones;que garantice unsistema de acceso a la creación de una historia digna, en continua reescritura, y que esté alerta, y alerte, de discriminaciones y relaciones de poder. En definitiva, lo que pretende este artículo es perder su vigencia. 


\section{Referencias}

Berger, John. (1972). 2008. Ways of Seeing. London:Penguin Books

Constenla, Tereixa. 2013. "Los museos las prefieren monas" .

http://sociedad.elpais.com/sociedad/2013/12/01/actualidad/1385921483_501196.html

Cordero Reiman, Karen y Sáenz, Inda. 2012. Crítica Feminista en la Teoría e Historia del Arte (Compilación). Ciudad de México : FONCA, Univ. Iberoamericana

Nochlin, Linda.1971. Art and Sexual Politics: Why Have There Been No Great Women Artists?. Ed. Thomas B. Hess y Elizabeth C. Baker. New York: Macmillan

Mendez, Lourdes. 2007. "Narrativas visuales feministas sobre la diferencia sexual: cuerpos, sexos, género y sexualidad".En Kiss Kiss Bang Bang : 45 años de arte y feminismo. VVAA. Catálogo de exposición. Ed. Xabier Arakistain. 41-53.Bilbao: Museo de Bellas Artes.

Phelan, Peggy. 2005.Art and Feminism. Ed. Helena Reckitt. New York:Pahidon

Pollock, Griselda. 2008. "Desde las intervenciones feministas hasta los efectos feministas en las historias del arte. Análisis de la virtualidad feminista y de las transformaciones estéticas del trauma". En Producción artística y teoría feminista del arte: nuevos debates I. VVAA. Ed.Xabier Arakistain y Lourdes Méndez.42-63. Vitoria: CC Montehermoso

Pollock, Griselda. 2007. "Feminidad, modernidad, representación". En Kiss Kiss Bang Bang : 45 anõs de arte y feminismo.VVAA.Catálogo de exposión. Ed. Xabier Arakistain. 22-35. Bilbao: Museo de Bellas Artes

Pollock, Griselda. 2002. "¿Puede la Historia del Arte sobrevivir al Feminismo?"Estudios Online. http://www.estudiosonline.net/texts/pollock.htm

(Artículo recibido 30-04-17; aceptado 03-07-2017) 